\title{
O estímulo à criatividade no contexto universitário
}

\author{
Eunice M.L. Soriano de Alencar \\ Universidade Católica de Brasília
}

\begin{abstract}
Resumo
O estudo examinou a extensão em que diferentes aspectos relativos à criatividade têm sido estimulados no contexto universitário, bem como a percepção, por parte de universitários, do nível de sua criatividade, do de seus colegas e professores. Quatrocentos e vinte e oito estudantes de universidades pública e particular participaram do estudo, respondendo a um inventário de incentivo à criatividade no contexto universitário, além de avaliar o nível de sua criatividade e o de seus professores e colegas. Os resultados apontam o pouco incentivo a distintos aspectos da criatividade por parte de professores universitários. Observou-se também que os universitários julgaram-se e a seus colegas como significativamente mais criativos do que os seus professores. Os resultados apontam a necessidade de maior atenção à expressão da cri atividade no contexto universitário.

Palavras-chave: criatividade, universidade, ensino.
\end{abstract}

\section{Motivation of creativity at university context}

\section{Summary}

The study investigated the degree to which different aspects related to creativity have been fostered by university teachers, as well as university students' evaluation of their own level of creativity, their teachers' level and their colleagues' level of creativity. Four hundred and twenty eight university students answered an inventory of incentive of creativity in the university context. They also evaluated their own level of creativity, and their teachers' and colleagues' level of creativity. The results called attention to the low degree of incentive to different aspects of creativity by university teachers. Students judged themselves and their colleagues as significantly more creative than their teachers. The results suggest the need of more atention to the development of creativity in the university context.

Key words: creativity, university, teaching.

Uma análise das pesquisas sobre criatividade no contexto educacional indica que a grande maioria dos estudos tem sido realizada com amostras de professores e alunos do primeiro grau e em menor escala com alunos do segundo grau. Observa-se uma enorme carência de pesquisas nesta área com amostras de estudantes/professores universitários. Nota-se ainda que os estudos no contexto universitário têm se restringido sobretudo à investigação de métodos e técnicas voltados para o desenvolvimento de habilidades criativas.

Um número significativo de pesquisas foi feito, por exemplo, por Parnes e colaboradores, com amostras de estudantes universitários, com vistas a investigar: (a) os efeitos de um programa semestral na estimulação deliberada da criatividade; (b) os efeitos nas habilidades criativas de um curso programado, utilizado independentemente ou com instrutores; (c) a efetividade de diferentes estratégias de resolução criativa de problemas. Uma síntese destas investigações é apresentada por Parnes (1987), apontando ganhos decorrentes de participação no programa. Estudos similares investigando os efeitos de um workshop de criatividade nas habilidades criativas de universitários foram feitos por Glover $(1977,1980)$, e no Brasil, Alencar e Virgolim (1990) investigaram também os efeitos de um programa de criatividade nas habilidades criativas de estudantes universitários. Outros estudos com amostras de universitários foram feitos por Amabile (1983), a respeito dos efeitos da expectativa de avaliação na criatividade. 
Há ainda alguns poucos estudos apontando a necessidade de se cultivar as habilidades criativas de universitários. Neste sentido, Shallcross e Gawienowski (1989) descrevem um simpósio sobre criatividade ocorrido na Universidade de Massachusetts, Estados Unidos, para chamar a atenção para a relevância da criatividade no contexto universitário e apontar maneiras de cultivá-la. Várias questões foram discutidas naquele simpósio especialmente com relação ao reconhecimento e desenvolvimento do potencial para o trabalho criativo e maneiras de se facilitar a expressão da energia criativa do universitário, aspectos estes também abordados em um outro simpósio subvencionado pela Fundação Norte-Americana de Ciências em 1987. Um estudo foi ainda conduzido por McDonough e McDonough (1987) sobre cursos de criatividade oferecidos por 76 universidades e outros por Baloche, Montgomery, Bull e Salyer (1992) e Montgomery, Bull e Baloche (1992), que descreveram os objetivos de cursos, bem como experiências, projetos e produtos exigidos em cursos de criatividade, em uma amostra de 147 professores de universidades norte-americanas.

Pesquisas relativas à extensão em que as habilidades criativas de estudantes universitários têm sido estimuladas pelo sistema e a percepção que os estudantes universitários têm de suas próprias habilidades criativas, de seus colegas e professores não foram encontradas na literatura especializada consultada. O que tem sido mais destacado na literatura é a responsabilidade da educação no desenvolvimento das habilidades criativas em todos os níveis de ensino (Guilford, 1968; Von Ardenne, 1990), bem como a necessidade de um planejamento deliberado pelo sistema educacional para o desenvolvimento das habilidades criativas (Isaksen, 1987, Renzulli, 1992; Slabbert, 1994). No Brasil, Rosas (1988) também ressalta o pouco incentivo à criatividade na universidade. Esta pesquisadora considera, por exemplo, que "é no terceiro grau onde menos se fala e pensa em criatividade. Excetuando-se as escolas e/ou departamentos de artes, parece que os demais professores têm muito mais o que fazer do que se preocupar com a imaginação, fantasia e criação" (p. 122), lembrando que é necessário que o professor universitário ultrapasse o papel de conservador e transmissor, para o de inovador, produtor, criador.

É o objetivo do presente artigo apresentar alguns dados obtidos pela autora relativos à extensão em que a criatividade vem sendo estimulada no contexto universitário, bem como a avaliação de universitários a respeito de suas próprias habilidades criativas, das habilidades criativas de seus colegas e professores. Compararam-se ainda estudantes do sexo masculino e feminino, de universidade pública e particular, de duas áreas (Humanas e Exatas), que se encontravam na primeira e segunda metade de sua formação acadêmica e que trabalhavam ou apenas estudavam nas variáveis de interesse - avaliação pelos alunos do estímulo à criatividade por parte de seus professores universitários e percepção do seu nível de criatividade, de seus professores e colegas.

\section{Método}

\section{Sujeitos}

A amostra foi constituída por 428 estudantes universitários. Deste total, 226 (52,8\%) eram alunos de uma universidade pública e 200 (46,7\%) de uma universidade particular, ${ }^{1}$ sendo que 232 (54,2\%) eram da área de Humanas (Comunicação e Pedagogia) e 194 (45,3\%) da área de Exatas

\footnotetext{
${ }^{1}$ Faltou informação sobre universidade e curso para dois sujeitos.
} 
(Matemática e Ciências da Computação). Cento e sessenta e três sujeitos (38,1\%) eram do sexo masculino e 265 (61,9\%) eram do sexo feminino. Da amostra total, 255 (59,6\%) informaram que também trabalhavam e 161 (37,6\%) que apenas estudavam (12 sujeitos deixaram de informar sobre este aspecto).

A idade média da amostra foi de 22,54 anos, com amplitude entre 17 e 47 anos. $74,6 \%$ da amostra tinha entre 17 e 23 anos. Duzentos e vinte e nove alunos (53,5\%) cursavam a primeira metade do curso e os demais (199 - 46,5\%) a segundo metade.

\section{Instrumento}

Utilizou-se um inventário para avaliar a percepção do estudante universitário quanto ao grau de incentivo a diferentes aspectos da criatividade por parte de seus professores. Esse inventário foi construído pela autora após constatar a inexistência de um instrumento que pudesse ser utilizado para a coleta de dados relativos à extensão em que diferentes aspectos relacionados à criatividade têm sido estimulados pelos professores universitários. Conforme descrito por Alencar (1995) e Alencar, Carvalho Meirelles e Pacheco (1993), a primeira etapa na elaboração do instrumento foi a construção de 23 itens relativos a diversas dimensões da criatividade, como traços de personalidade, pensamento criativo, metodologia de ensino e condições de aprendizagem. Estes itens foram elaborados com base na literatura relativa às diferentes dimensões da criatividade e condições favoráveis ao seu desenvolvimento, bem como no instrumento "Questionário de Avaliação da Docência", da autoria de Pasquali, Siqueira e Marques (1978).

Procedeu-se, então, a uma avaliação semântica dos itens construídos, seguindo-se da aplicação do instrumento em uma amostra de 210 estudantes universitários, com vistas a obter dados para a validação estatística do mesmo. A seguir, foi feito um estudo do conteúdo do instrumento, procedendo-se distintas análises fatoriais e análise de consistência interna, conforme descrito em Alencar (1995).

Seguem-se alguns dos itens do instrumento construído:

Os professores universitários, de modo geral:

- Levam o aluno a perceber e conhecer pontos de vista divergentes sobre o mesmo problema ou tema de estudo.

- Incentivam os alunos a fazer questões relativas aos temas estudados.

- Estimulam a curiosidade dos alunos através das tarefas propostas em suas disciplinas.

- Dão tempo aos alunos para pensar e desenvolver idéias novas.

- Cultivam nos alunos o gosto pela descoberta e pela busca de novos conhecimentos.

- Utilizam provas e avaliações que exigem do aluno apenas a reprodução de conteúdo dado em classe ou contido nos livros-textos (a pontuação deste item foi invertida para fins de análise).

Cada um desses itens era respondido em uma escala de cinco pontos que variava de discordo plenamente até concordo plenamente.

Os estudantes responderam também aos seguintes itens relativos a sua percepção do nível de sua própria criatividade, de seus colegas e professores:

Como você se classifica em uma escala de criatividade?

Como você classificaria o nível de criatividade de seus professores atuais? 
Como você classificaria o nível de criatividade de seus colegas atuais?

Esses itens foram respondidos em uma escala de sete pontos, variando de nada criativo (1) a extremamente criativo (7).

\section{Resultados}

Percepção dos estudantes universitários quanto ao estímulo à criatividade por parte de seus professores.

Em função do elevado número de variáveis independentes (tipo de universidade, área de curso, período de formação universitária, sexo e trabalho) optou-se por realizar uma análise de variância multivariada de três fatores tipo de universidade, área de curso e período de formação (cada uma com dois níveis) e outra de dois fatores - sexo e trabalho (cada uma também com dois níveis) para análise dos dados obtidos através do inventário.

Tabela 1. Média, desvio-padrão e valor $\mathrm{F}$ na escala que avaliava o incentivo à criatividade por parte dos professores universitários para estudantes da universidade pública e particular das áreas de Humanas e Exatas, da primeira e segunda metades do curso, do sexo masculino e feminino, que trabalhavam ou apenas estudavam

\begin{tabular}{|c|c|c|c|c|}
\hline Variáveis & Média & DP & $\mathrm{F}$ & $\mathrm{p}$ \\
\hline \multicolumn{5}{|c|}{ Tipo de universidade } \\
\hline Pública & 2,95 & 0,60 & 0,01 & n.s. \\
\hline Particular & 2,91 & 0,56 & & \\
\hline \multicolumn{5}{|l|}{ Área } \\
\hline Humanas & 2,93 & 0,57 & 0,43 & n.s. \\
\hline Exatas & 2,95 & 0,59 & & \\
\hline \multicolumn{5}{|l|}{ Período } \\
\hline Primeira metade & 3,06 & 0,59 & 21,53 & 0,000 \\
\hline Segunda metade & 2,79 & 0,54 & & \\
\hline \multicolumn{5}{|l|}{ Sexo } \\
\hline Masculino & 2,97 & 0,61 & 01,83 & n.s. \\
\hline Feminino & 2,91 & 0,56 & & \\
\hline \multicolumn{5}{|l|}{ Trabalho } \\
\hline Sim & 2,89 & 0,58 & 2,67 & n.s. \\
\hline Não & 3,01 & 0,58 & & \\
\hline
\end{tabular}

Pelos resultados apresentados na Tabela 1, observa-se que a única diferença significativa na escala foi obtida entre estudantes da primeira e última metade do curso - F $(1,364)=21,53$, $\mathrm{p}=0,000$. Os estudantes dos primeiros semestres avaliaram os seus professores universitários como propiciando maior incentivo à promoção da criatividade em suas disciplinas comparativamente aos dos últimos semestres.

A análise de variância realizada indicou ainda uma interação entre as variáveis tipo de universidade e área - F $(1,364)=8,80, \mathrm{p}=0,003$. Para facilitar a compreensão desta interação, apresentamos na Tabela 2 as médias obtidas pelos sujeitos da universidade pública e particular, dos cursos de Humanas e Exatas na escala. 
Tabela 2. Média obtida pelos sujeitos da universidade pública e particular das áreas de Humanas e Exatas na escala de avaliação do incentivo à criatividade pelos professores universitários

\begin{tabular}{|c|c|c|}
\hline \multirow{2}{*}{ Universidade } & \multicolumn{2}{|c|}{ Área } \\
\cline { 2 - 3 } & Humanas & Exatas \\
\cline { 2 - 3 } & Média & Média \\
\hline Pública & 3,00 & 2,87 \\
\hline Particular & 2,80 & 3,01 \\
\hline
\end{tabular}

Como pode ser observado pelas médias apresentadas na Tabela 2, os sujeitos dos cursos de Humanas da Universidade pública e de Exatas da universidade particular avaliaram os seus professores universitários como apresentando maior incentivo à criatividade, comparativamente aos estudantes dos cursos de Humanas da universidade particular e de Exatas da universidade pública.

Auto-avaliação do nível de criatividade e do nível de criatividade de seus professores e colegas por parte dos estudantes universitários.

Na Tabela 3, são apresentados a média, desvio-padrão e valor F relativos à auto-avaliação do nível de criatividade por parte dos estudantes da universidade pública e particular, da área de Humanas e Exatas, do sexo masculino e feminino, da primeira e segunda metades de sua formação universitária e que trabalhavam ou apenas estudavam. Como pode ser observado nesta Tabela, os sujeitos do sexo masculino se avaliaram como significativamente mais criativos do que os do sexo feminino $-F(1,406)=24,55, p=0,0000$. Observou-se ainda que os sujeitos dos últimos semestres se avaliaram como significativamente mais criativos do que os dos primeiros semestres - F $(1,409)$ $=3,98, \mathrm{p}=0,0467)$, o mesmo ocorrendo com os estudantes que trabalhavam, que também se avaliaram como significativamente mais criativos do que aqueles que apenas estudavam - $\mathrm{F}(1,406)$ $=4,08, \mathrm{P}=0,0440$.

Tabela 3. Média, desvio-padrão e valor F na auto-avaliação do nível de criatividade por parte dos estudantes da universidade pública e particular, das áreas de Humanas e Exatas, do sexo masculino e feminino, da primeira e segunda metades do curso e que trabalhavam ou apenas estudavam

\begin{tabular}{|c|c|c|c|c|}
\hline Variáveis & Média & DP & F & $\mathrm{p}$ \\
\hline \multicolumn{5}{|c|}{ Tipo de universidade } \\
\hline Pública & 3,58 & 1,16 & 1,87 & n.s. \\
\hline Particular & 3,74 & 1,24 & & \\
\hline \multicolumn{5}{|l|}{ Área } \\
\hline Humanas & 3,60 & 1,13 & 0,71 & n.s. \\
\hline Exatas & 3,70 & 1,27 & & \\
\hline \multicolumn{5}{|l|}{ Sexo } \\
\hline Masculino & 4,01 & 1,28 & 24,55 & 0,000 \\
\hline Feminino & 3,43 & 1,09 & & \\
\hline \multicolumn{5}{|c|}{ Período de curso } \\
\hline Primeira metade & 3,54 & 1,21 & 3,98 & 0,0467 \\
\hline Segunda metade & 3,78 & 1,18 & & \\
\hline \multicolumn{5}{|l|}{ Trabalho } \\
\hline Sim & 3,75 & 1,23 & 4,08 & 0,0440 \\
\hline Não & 3,50 & 1,13 & & \\
\hline
\end{tabular}


Quanto à avaliação do nível de criatividade de seus professores, a única diferença significativa obtida foi a observada entre estudantes do sexo masculino e feminino - $F(1,410)=9,93, p=$ 0,0017 , tendo os primeiros considerado os seus professores como mais criativos do que os estudantes do sexo feminino. Na Tabela 4, os resultados obtidos com relação a este aspecto (média, desvio-padrão e valor F) são apresentados.

Tabela 4. Média, desvio-padrão e valor F na avaliação do nível de criatividade de seus professores por parte dos estudantes da universidade pública e particular, das áreas de Humanas e Exatas, do sexo masculino e feminino, da primeira e segunda metades do curso, que trabalhavam ou apenas estudavam

\begin{tabular}{|c|c|c|c|c|}
\hline Variáveis & Média & DP & $\mathrm{F}$ & $\mathrm{p}$ \\
\hline \multicolumn{5}{|c|}{ Tipo de universidade } \\
\hline Pública & 2,86 & 1,05 & 0,09 & n.s. \\
\hline Particular & 2,89 & 1,17 & & \\
\hline \multicolumn{5}{|l|}{ Área } \\
\hline Humanas & 2,79 & 1,06 & 2,43 & n.s. \\
\hline Exatas & 2,96 & 1,16 & & \\
\hline \multicolumn{5}{|c|}{ Sexo } \\
\hline Masculino & 3,08 & 1,20 & 9,93 & 0,0017 \\
\hline Feminino & 2,74 & 1,03 & & \\
\hline \multicolumn{5}{|c|}{ Período de curso } \\
\hline Primeira metade & 2,82 & 1,11 & 1,14 & n.s. \\
\hline Segunda metade & 2,94 & 1,10 & & \\
\hline \multicolumn{5}{|l|}{ Trabalho } \\
\hline Sim & 2,91 & 1,10 & 0,95 & n.s. \\
\hline Não & 2,80 & 1,12 & & \\
\hline
\end{tabular}

Os resultados relativos à avaliação do nível de criatividade dos colegas são apresentados na Tabela 5. Como pode ser observado nesta Tabela, os estudantes da universidade particular consideraram os seus colegas como significativamente mais criativos do que os de universidade pública - $\mathrm{F}(1,406)=10,58, \mathrm{p}=0,0012$. Não foram observadas diferenças significativas nesta avaliação entre estudantes das áreas de Humanas e Exatas, do sexo masculino e feminino, da primeira e segunda metades do curso, e que trabalhavam ou apenas estudavam. 
Tabela 5. Média, desvio-padrão e valor F na avaliação do nível de criatividade dos colegas por parte dos estudantes da universidade pública e particular, das áreas de Humanas e Exatas, do sexo masculino e feminino, da primeira e segunda metades do curso, que trabalhavam ou apenas estudavam

\begin{tabular}{|c|c|c|c|c|}
\hline Variáveis & Média & DP & $\mathrm{F}$ & $\mathrm{p}$ \\
\hline \multicolumn{5}{|c|}{ Tipo de universidade } \\
\hline Pública & 3,56 & 1,03 & 10,58 & 0,0012 \\
\hline Particular & 3,92 & 1,20 & & \\
\hline \multicolumn{5}{|l|}{ Área } \\
\hline Humanas & 3,73 & 1,12 & 0,0004 & n.s. \\
\hline Exatas & 3,73 & 1,13 & & \\
\hline \multicolumn{5}{|l|}{ Sexo } \\
\hline Masculino & 3,77 & 1,20 & 0,29 & n.s. \\
\hline Feminino & 3,71 & 1,08 & & \\
\hline \multicolumn{5}{|c|}{ Período de curso } \\
\hline Primeira metade & 3,76 & 1,13 & 0,21 & n.s. \\
\hline Segunda metade & 3,71 & 1,12 & & \\
\hline \multicolumn{5}{|l|}{ Trabalho } \\
\hline Sim & 3,72 & 1,15 & 0,005 & n.s. \\
\hline Não & 3,73 & 1,10 & & \\
\hline
\end{tabular}

Com base nos dados levantados quanto à avaliação de sua própria criatividade, da criatividade de seus professores e colegas pelos sujeitos que constituíram a amostra do presente estudo, procedeu-se também a uma comparação entre estes três aspectos pelos mesmos sujeitos no sentido de se verificar a extensão em que a amostra estudada se percebia como mais criativa ou menos criativa que seus professores e colegas. Na Tabela 6, são apresentados a média e o desvio-padrão obtidos para a avaliação do seu nível de criatividade, do nível de criatividade de seus professores e colegas para uma amostra de 406 sujeitos que responderam a estes itens.

Tabela 6. Média e desvio-padrão na avaliação do seu próprio nível de criatividade, do nível de criatividade de seus professores e colegas por parte da amostra

\begin{tabular}{|c|c|c|c|c|c|}
\hline \multicolumn{2}{|c|}{$\begin{array}{c}\text { Auto-avaliação } \\
\text { de criatividade }\end{array}$} & \multicolumn{2}{|c|}{$\begin{array}{c}\text { Avaliação criat. } \\
\text { professores }\end{array}$} & \multicolumn{2}{|c|}{$\begin{array}{c}\text { Avaliação criat. } \\
\text { colegas }\end{array}$} \\
\hline Média & DP & Média & DP & Média & DP \\
\hline 3,65 & 1,20 & 2,87 & 1,11 & 3,73 & 1,12 \\
\hline
\end{tabular}

O teste "t" de Student foi utilizado para análise das diferenças entre estas médias, tendo os resultados obtidos indicado que os sujeitos se perceberam como significativamente mais criativos do que os seus professores ( $\mathrm{t}=9,68, \mathrm{p}<0,001$ ), avaliando ainda os seus colegas como significativamente mais criativos do que os seus professores universitários $(t=11,03, p<0,001)$. Não foi significativa a diferença entre a avaliação do seu próprio nível de criatividade e a de seus colegas $(\mathrm{t}=0,98$, $\mathrm{p}-\mathrm{n} . \mathrm{s}$. $)$. 


\section{Discussão}

Os resultados obtidos no presente estudo chamam a atenção para o fato de que, de modo geral, os estudantes universitários consideram que há pouco incentivo a distintos aspectos da criatividade por parte de seus professores. Tais resultados coincidem com o que foi destacado anteriormente por Rosas (1988), que chamou a atenção para o baixo interesse pelo desenvolvimento da criatividade por parte do corpo docente universitário.

Observou-se ainda que, na escala de incentivo à criatividade, foram os alunos dos primeiros semestres os que avaliaram os seus professores como promovendo melhores condições à expressão da criatividade, comparativamente aos dos últimos semestres. Possivelmente, este resultado pode ser explicado pela diferença de metodologia de ensino mais comumente observada entre o segundo e o terceiro graus. No segundo grau, há uma preocupação grande com a reprodução do conhecimento, vista como necessária à preparação para o vestibular. Em estudo anterior com uma amostra de segundo grau (Alencar, Collares, Dias \& Julião, 1993), os alunos informaram, por exemplo, que o programa de sua escola não favorecia o desenvolvimento e expressão de suas habilidades criativas. Por outro lado, na universidade, a tendência é dar maior incentivo ao estudo independente, a exigir do aluno maior iniciativa, a se fazer uso de formas de avaliação que não aquelas que exigem apenas a reprodução do conteúdo dado em classe ou contido nos livros-textos, aspecto este mais evidente para os alunos dos primeiros semestres de sua formação universitária.

Ao responderem à escala de incentivo à criatividade, observou-se também uma diferença entre estudantes da área de Exatas e Humanas das duas universidades, constatando-se que os estudantes da área de Humanas da universidade pública e de Exatas da universidade particular apontaram os seus professores como apresentando maior incentivo à criatividade do que os demais. Era nossa expectativa de que, caso esta diferença ocorresse, ela seria a favor dos estudantes da universidade pública, independentemente de sua área de curso. Isto porque o número de alunos por turma na universidade pública onde os dados foram coletados era menor, muitos de seus professores eram também pesquisadores, com uma formação de um nível mais elevado em termos de pósgraduação, o que poderia estar refletindo em sala de aula, no espaço para discussão de idéias divergentes, no tipo de pergunta que se faz ao aluno, na promoção de um ambiente de maior respeito e aceitação por novas idéias, no estímulo à independência e iniciativa e em uma maior inovação no processo de ensino. Isto, entretanto, não ocorreu, sugerindo que especialmente os professores da área de Exatas da universidade pública estavam menos equipados para prover um ambiente propício ao desenvolvimento e expressão da criatividade.

Quando avaliaram o seu próprio nível de criatividade, observou-se que estudantes do sexo masculino, da segunda metade do curso e que trabalhavam consideraram-se como mais criativos do que os do sexo feminino, da primeira metade e que somente estudavam. É notório que, em pesquisas com amostras de alunos do primeiro grau (Alencar, Fleith, Shimabukuro \& Nobre, 1987; Rodrigues \& Alencar, 1983, Virgolim \& Alencar, 1993), utilizando-se testes de pensamento criativo, foi observado um desempenho superior por parte de amostras do sexo feminino.

O fato de o universitário do sexo masculino perceber-se como mais criativo do que a amostra feminina possivelmente reflete uma dimensão do autoconceito, que merece ser mais bem explorada em pesquisas futuras. Há vários estudos chamando a atenção para o papel da percepção e avaliação de si mesmo na orientação e controle do próprio comportamento (Markus \& Wurf, 1987). Esses estudos sugerem que se o indivíduo se percebe e se avalia como competente, capaz e criativo, ele 
tende a ter mais confiança em expressar idéias e em exibir comportamento criativo. Por outro lado, se o indivíduo se percebe como incapaz e não criativo, esta percepção irá refletir em suas ações, limitando as possibilidades de uma expressão mais plena de seu potencial e talento.

Características de personalidade mais cultivadas entre pessoas do sexo masculino e feminino na sociedade ocidental também poderiam ser um fator a contribuir para explicar os resultados aqui encontrados quanto à diferença entre sexos na avaliação do próprio nível de criatividade. Alguns traços, como independência, iniciativa e mesmo autoconfiança são tradicionalmente mais cultivados entre pessoas do sexo masculino, e talvez contribuam para uma percepção de si mesmo como mais criativo por parte da amostra masculina.

A diferença entre estudantes que trabalhavam ou apenas estudavam na medida de avaliação do seu nível de criatividade a favor dos que também trabalhavam possivelmente pode ser atribuída ao fato de que conciliar o estudo com o trabalho exija um exercício permanente de criatividade, o que se refletiu nesta avaliação.

Observou-se, no estudo, que os universitários percebiam, de modo geral, os seus professores como pouco ou muito pouco criativos. Eles foram avaliados como menos criativos do que os seus colegas e do que eles próprios. Possivelmente esta percepção reflete o comportamento do professor em sala de aula que denota pouca criatividade. Tal resultado, que também foi observado em estudo anterior com uma amostra de estudantes do $2^{\circ}$ grau (Alencar, 1993), sugere a pouca ênfase que se dá à criatividade por parte dos professores universitários. Estes estariam mais preocupados com 0 seu papel de transmissor de informação, como já havia sido assinalado anteriormente por Rosas (1988). Consideramos de relevância maior, neste final de milênio, que o professor universitário esteja mais atento ao desenvolvimento da capacidade do futuro profissional de pensar de forma criativa e inovadora, algo indispensável neste momento da História marcado pela mudança, pela incerteza, por um progresso sem precedente, e por uma necessidade permanente do exercício da própria capacidade de pensar. Isto indubitavelmente está a exigir novas práticas de ensino, constituindo-se no desafio aos educadores de passarem a exercer o papel de catalisadores do potencial criativo de cada aluno. Esta nova postura certamente resultaria em um menor desperdício de talento e potencial humano, como vem ocorrendo em conseqüência das possibilidades limitadas oferecidas ao desenvolvimento e expressão da criatividade no ambiente universitário.

\section{Referências}

Alencar, E. M. L. S. (1993). Como percebem os alunos o seu nível de criatividade, o de seus colegas e professores. Trabalho apresentado no Grupo de Trabalho "Contribuições da Psicologia para a superação do fracasso escolar", na quinta reunião da ANPEPP, Caxambu, Minas Gerais.

Alencar, E. M. L. S. (1995). Developing creative abilites at the university level. European Journal for High Ability, 6, 82-90.

Alencar, E. M. L. S., Carvalho, C. C., Meirelles, 1. B., \& Pacheco, P. R. C. (1993). Construção de uma escala para avaliação da percepção de estudantes quanto ao estímulo à criatividade no sistema universitário. Anais do XXIII Reunião Anual de Psicologia da Sociedade Brasileira de Psicologia. Ribeirão Preto: Sociedade Brasileira de Psicologia.

Alencar, E. M. L. S., Collares, K., Dias, L. \& Julião, S. (1993). Efeitos a curto e médio prazos de um programa de treinamento de criatividade em estudantes do ensino de segundo grau. Anais da XXIII Reunião Anual da Sociedade Brasileira de Psicologia. Ribeirão Preto: Sociedade Brasileira de Psicologia.

Alencar, E. M. L. S., Fleith, D. S., Shimabukuro,L. A. \& Nobre, M. A. (1987). Efeitos de um programa de treinamento de criatividade para professores do ensino de primeiro grau nas habilidades de pensamento 
criativo do aluno. Revista Interamericana de Psicologia, 21, 56-71.

Alencar, E. M. L. S., \& Virgolim, A. M. (1990). Desenvolvimento de habilidades criativas de estudantes universitários. Anais da XX Reunião Anual da Sociedade de Psicologia de Ribeirão Preto (p. 149). Ribeirão Preto: Sociedade de Psicologia de Ribeirão Preto.

Amabile, T. M. (1983). The Social Psychology of creativity. Nova York : Springer.

Baloche, L., Montgomery, D., Bull, K. S. \& Salyer, B. K. (1992). Faculty perceptions of college creativity courses. Journal ofCreative Behavior, 26,222-227.

Glover, J. A. (1977). An investigation of the relationship offour components of creativity to locus of control. Social Behavior \& Personality, 4, 257-260.

Glover, J. A. (1980). A creativity-training program workshop: Short-term, long-term \& transfer effects. The Journal of Genetic Psychology, 136, 3-16.

Guilford, 1. P. (1968). Intelligence, creativity and their educational implications. San Diego, CA: Knapp.

Isaksen, S. G. (1987). Frontiers of creativity research. Beyond the basics. Buffalo, N.Y.: Bearly.

Markus, H. \& Wurf, E. (1987). The dynamic selfconcept: A social psychological perspective. Annual Review of Psychology, 38, 299-337.

McDonough, P. \& McDonough, B. (1987). A survey of American colleges and universities on the conducting of formal courses in creativity. Journal ofCreative Behavior, 21, 271-282.

Montgomery, D., Bull, K. S. \& Baloche, L. (1992). College level creativity course content. Journal o fCreative Behavior, 26,228-235.

Parnes, S. 1. (1987). The Creative Studies Project. Em S. G. Isaksen (Ed.), Frontiers of creativity research. Beyond the basics (pp. 156-188). Buffa10, N.Y.: Bearly.

Pasquali, L., Siqueira, M. M. M., \& Marques, S. M. (1978). Questionário de Avaliação da Docência. Brasília: Universidade de Brasília.

Renzulli, J. S. (1992). A general theory for the development of creative productivity in young people. Em F. 1. Mõnks, \& W. Peters (Eds.). Talent for the future (pp.51-72). Assen/Maastricht, The Netherlands: Van Gorcum.

Rodrigues, C. J. S. \& Alencar, E. M. L. S. (1983). Um estudo de pensamento criativo em alunos do primeiro grau. Arquivos Brasileiros de Psicologia, 35, 113-122.

Rosas, A. (1988). Universidade e criatividade. Anais do XVII Seminário Nacional sobre Superdotados (pp. 121-124). Rio de Janeiro: SENAI.

Shallcross, D. 1. \& Gawienowski, A. M. (1989). Top experts address issues on creativity gap in higher education. Journal of Creative Behavior, 23,75-84.

Slabbert, 1. A. (1994). Creativity in education revisited: Reflection in aid of progression. Journal of Creative Behavior, 28,60-69.

Virgolim, A. M. R. \& Alencar, E. M. L. S. (1993). Habilidades de pensamento criativo entre alunos de escolas abertas, intermediárias e tradicionais. Psicologia: Teoria e Pesquisa, 9, 601-610.

Von Ardenne, M. (1990). Facilitating the development of talents. European Journal for High Ability, 1, 127135. 